\title{
Mesoscale damage analysis of needle-punched carbon/carbon composite considering randomness of inherent defects
}

\author{
Meng Han ${ }^{\mathrm{a}, \mathrm{b}}$, Chuwei Zhou ${ }^{\mathrm{a}, *}$, Vadim V. Silberschmidt ${ }^{\mathrm{b}}$ \\ ${ }^{a}$ State Key Laboratory of Mechanics and Control of Mechanical Structures, Nanjing University of Aeronautics and Astronautics, Nanjing, 210016, PR China \\ ${ }^{\mathrm{b}}$ Wolfson School of Mechanical, Electrical and Manufacturing Engineering, Loughborough University, Loughborough, Leicestershire, LE11 3TU, UK
}

\begin{abstract}
A B S T R A C T
Needle-punched carbon/carbon composites (NP C/Cs) are widely used in aerospace applications thanks to their good high-temperature mechanical properties with relatively low cost. To improve their out-plane stiffness and strength, short-cut fibres are introduced into interlayers between plies using a needle-punching technology. This could result in some defects that decrease their in-plane stiffness and strength. Circle-arc beam elements and extended spring elements are proposed in this research to model mechanical performances of punched fibre bundles, isotropic pyrocarbon matrix and short-cut carbon fibre felts in NP C/Cs, respectively. Aided by this beam-spring finite element model, the impacts from features of mesoscopic defects, e.g. irregularity of distribution of local imperfection in planar fiber bundle caused by punching process, to mechanical properties of NP $\mathrm{C} / \mathrm{Cs}$ are focused in this study. The ranges of bending modulus and bending strength for a NP C/C are estimated considering the randomness of these defects.
\end{abstract}

Keywords:

Needle punched carbon/carbon composites

Mesoscale mechanical model

Statistical analysis

Finite element analysis

\section{Introduction}

Needle-punching is a widely used low-cost technology to produce quasi three dimensional fibre-reinforced composites, especially carbon/ carbon composites (C/Cs). Needle-punched C/Cs (NP C/Cs) demonstrate excellent mechanical properties in high temperature environment, so they have been found many applications in fields of aerospace engines and brake systems of airplanes and race cars [1].

Efforts, mainly experimental ones, have been spent on well understanding the mechanical performance and failure mechanism of NP C/ Cs. For instance, the quasi-static and high-strain-rate compression experiments were performed on 3D NP C/Cs at room and elevated temperatures, the results showed that the dynamic compression strength and modulus increased with the increase of temperature (up to $300{ }^{\circ} \mathrm{C}$ ), while the static ones decreased significantly $[2,3]$. The bending fracture behavior of NP C/C was found closely relevant to both its microstructure and pre-fatigue loads which it had experienced [4]. Its loaddeflection curves were found exhibiting linear elasticity and brittle fracture below $400{ }^{\circ} \mathrm{C}$ and demonstrating obvious toughness and plastic failure mode above $500{ }^{\circ} \mathrm{C}$ [5], in addition, the bending properties were affected greatly by the fiber architecture, the plain structure was superior to the non-woven structure [6]. Furtherly, its bending strength was influenced by frequency of high-temperature treatment [7].

Due to the random distribution of needling introduced defects, the properties of NP C/C are obviously diverse and exactly representing its microstructures is difficult, thus to develop a mechanical property model of this composite theoretically and numerically characterizing these uncertainties are still open issues. Xie [8,9] employed random functions to describe disorder distributions of needling defects which were believed chiefly responsible for the scattering of mechanical properties of this composite. Zhang and Zhou [10] elaborated a computational approach to predict the strength of NP C/Cs, in which the mesoscopic structure of the composite was classified to five typical unit cells based on optical microscope observation. Yu and Zhou [11] modelled a 3D mesoscopic FE approach of a NP C/C which was reconstructed from micro-CT images and was used to investigate the transformation of dominated damage mechanisms in progressive damage process. Combining shear-lag model and finite element model, Jia [12] investigated the discrepancy between local residual stress concentrations caused by ordered and disordered patterns of needlepunching, respectively.

In this paper, a mesoscale beam-spring combined model is developed to analyze the mechanical performance of NP C/Cs. The beam and spring elements characterize fibre bundle and matrix-rich areas (and the short cut carbon felt layer) in NP C/Cs, respectively. Degrees of freedom of this proposed model are greatly reduced compared with 3D meso FEM model with 3D brick element [11]. Therefore it offers a mesoscopic analysis method to represent the complicated mesoscopic

\footnotetext{
* Corresponding author.

E-mail address: zcw@nuaa.edu.cn (C. Zhou).
} 


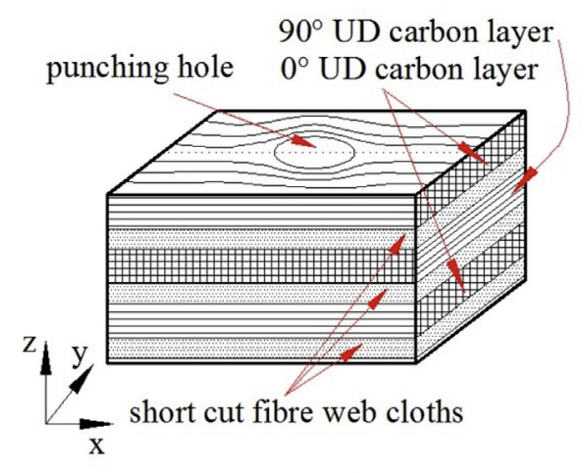

(a) ply pattern and pinhole

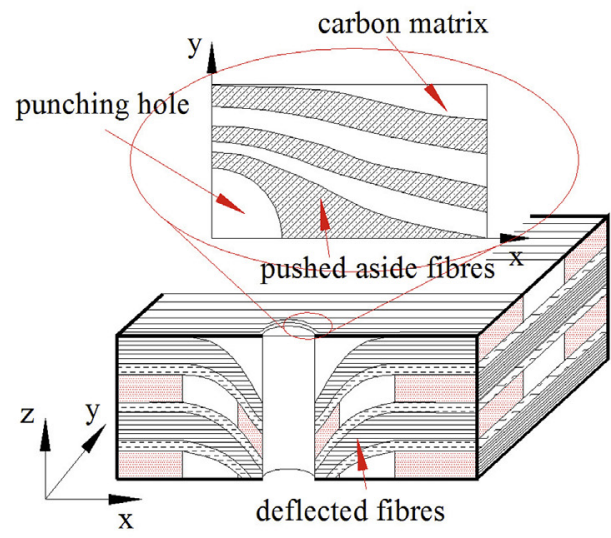

(b) deflected and pushed aside fibre bundles

Fig. 1. Schematic diagram of mesoscale structure of NP C/C.

features in NP C/Cs in detail with relatively low computing cost. Through randomly setting mesoscopic structure parameters in the proposed model, such as punching holes' positions and stagger distances between fiber bundles in different plies, the variation ranges of flexible modulus and strength of a NP C/C are estimated.

\section{Mesoscopic structure of NP C/Cs}

The studied NP C/C was formed by $0^{\circ}$ and $90^{\circ}$ unidirectional (UD) $12 \mathrm{~K}$ T700 carbon laminas with short-cut fibre felt cloths sandwiched between them, as schematically shown in Fig. 1(a). In the region where local structure was not distorted by punching hole, the warp (or weft) density was 4.17 bundles/cm and width of each bundle was $1.5 \mathrm{~mm}$. The average thicknesses of UD and felt plies were measured $0.42 \mathrm{~mm}$ and $0.08 \mathrm{~mm}$ in unpunched area respectively.

In needle-punching process, planar fibre might be pushed aside in intralaminar (Figs. 1-2(a)), or deflected in thickness direction (Figs. 1(b)-2(b)) or even broken.

For achieving designed punching density, the preform of NP C/Cs is usually punched several times. Double-punching is a common process in which the preform is firstly needle punched, rotated by $90^{\circ}$ and then punched again. Dimensions and pattern of the least periodic needle arrangement in needle board used for manufacturing the NP C/C studied in this paper are shown in Fig. 3(a), and Fig. 3(b) is its $90^{\circ}$ rotation. After twice punching, distribution pattern of pinhole is repeated within a scope of $24 \mathrm{~mm} \times 24 \mathrm{~mm}$ region (Fig. 3(c)), in other words this region contains all the typical punching features in the composite. Hence, it can be employed as a representative volume element (RVE) for mesoscale mechanical modelling.

\section{A simplified numerical mesoscopic mechanics model of NP C/Cs}

\subsection{Circular-arc beam element and extended spring element}

An elaborate mechanical model of NP C/Cs should include at least one RVE (see Fig. 3(c)) for each ply and so rather huge computational cost is expected. To make parametric analysis of these composite feasible, a simplification of introducing two types of one-dimensional elements were suggested to represent various component phases in NP $\mathrm{C} / \mathrm{Cs}$, instead of using 3D brick element. The first element, named circular-arc beam element (CABE), contains two nodes, each with $6^{\circ}$ of freedom (DOFs), as shown in Fig. 4(a). When the element's curvature approaches zero, the CABE degenerates into an ordinary straight beam element (DCABE). CABE and DCABE represent fiber bundles which are the main in-planar load carriers in NP C/Cs.

The second type of element is an extended spring element (ESE) that contains two nodes, each with three DOFs (Fig. 4(b)). It was employed to model matrix-rich area and short cut fibre felt layer which bound fiber bundle as an integrate body. The detailed stiffness matrixes of CABE and ESE can be found in our previous work [13].

\subsection{Discretization of the proposed NP C/Cs' mechanical model}

The schematic mesoscopic structure of NP C/C is illustrated in Fig. 5(a). One fibre bundle in a cloth is modelled with several adjacent CABEs (or DCABEs) as shown in Fig. 5(b), so different defects introduced by punching can be represented through setting various inand inter-planar curvatures (or breakage) on these beam elements according to their damage degree. Fig. 6(a) illustrates the various possible

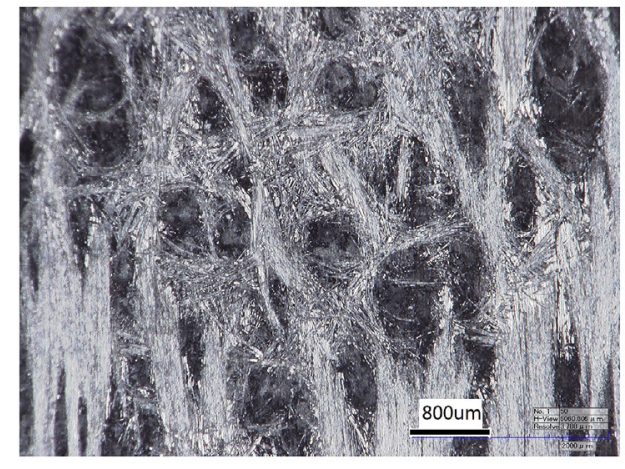

(a) pushed aside fibres

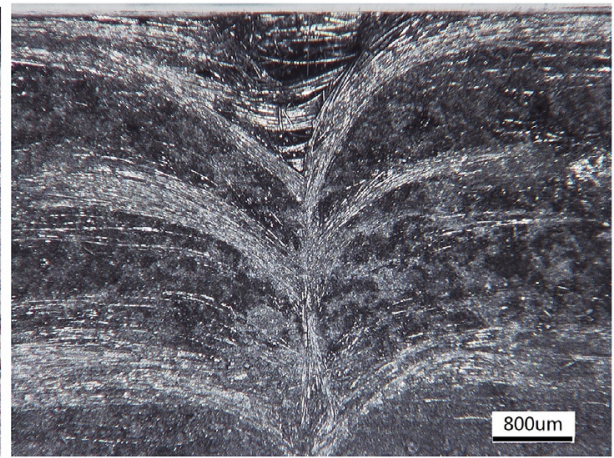

(b) deflected and broken fibres

Fig. 2. Mesoscopic photos of structure of NP C/C. 


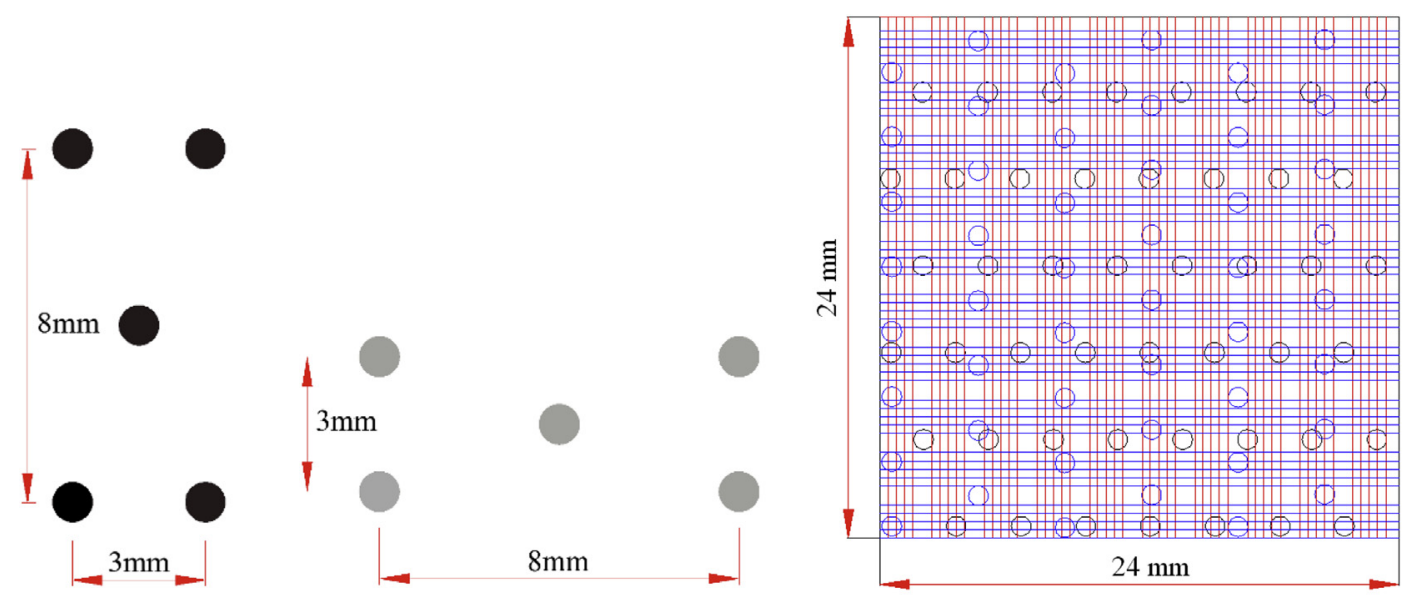

(a)

(b)

(c)

Fig. 3. Distribution of pinholes in NP C/C: (a) least periodic arrangement of needle in needle board; (b) $90^{\circ}$ rotation of the least periodic needle pattern; (c) distribution of needle holes in RVE region.

needle punching positions. A needle hole might locate entirely in fiber bundle or in matrix rich region, it also might partly in fibre bundle and partly in matrix region, holes can separate, close contact or even overlap partly. For the case a beam element lying within a pinhole area, if it locates just at central point of pinhole, it is considered deflecting through the felt ply to the next cloth ply without in-planar off-axis, see Fig. 6(b). Otherwise the beam element is expected deflecting off-planarly to the next cloth ply and deviating in-planarly to the pinhole edge as well (also see Fig. 6(b)). For the scenario of a beam element locating in the overlapped area of two pinholes which is introduced in double punching-process, this element is proposed broken at the maximum deflection point (see Fig. 6 (a)), this assumption reasons from the fact that the breakage possibility of a fibre increases if experienced twice punching. It should be noted that the position deviations of fibre bundles in different cloths with the same fibre orientation are set in the model, as the space $d_{1}$ and $d_{2}$ shown in Fig. 5(a). Fiber bundles, or fiber bundle beams, are connected by ESEs forming an integrate composite. There three types of ESEs are classified in the proposed model of NP C/ $\mathrm{Cs}$, i.e. the bind between CABEs (or DCABEs) belonging to the same fibre bundle is named ESE-fb; the rich pyrocarbon matrix region between neighbouring fibre bundles in same cloth ply is noticed as ESE-m; the inter-cloth cohesion which denotes ESE-fl. All these ESEs are illustrated in Fig. 5(b). It should be indicated that the ESEs at punching holes are modified from the three basic ESEs above depend upon where the punching hole locates.

\subsection{Equivalent mechanical property of $C A B E$}

The longitudinal properties of fibre bundle are represented by CABE (or DCABEs). The cross section of fibre bundle is idealized as rectangular, thus the cross section of CABE (or DCABE) is also rectangular with height of $h_{f b}$ and width of $w_{f b} / N_{b}$, where $N_{b}$ is the number of CABE (or DCABE) discretized in one fibre bundle. The equivalent longitudinal modulus of CABE is estimated by mixture law:

$E_{11}=c_{f b} E_{f 11}+\left(1-c_{f b}\right) E_{m}$

where, $E_{f 11}$ and $E_{m}$ are longitudinal modulus of fibre and modulus of carbon matrix, respectively, $c_{f b}$ is the volume fraction of fibre in fibre bundle. The detailed descriptions of stiffness matrix, local axial/ bending combined stresses as well as fibre directional damage model of CABE can be found in our previous work [13].
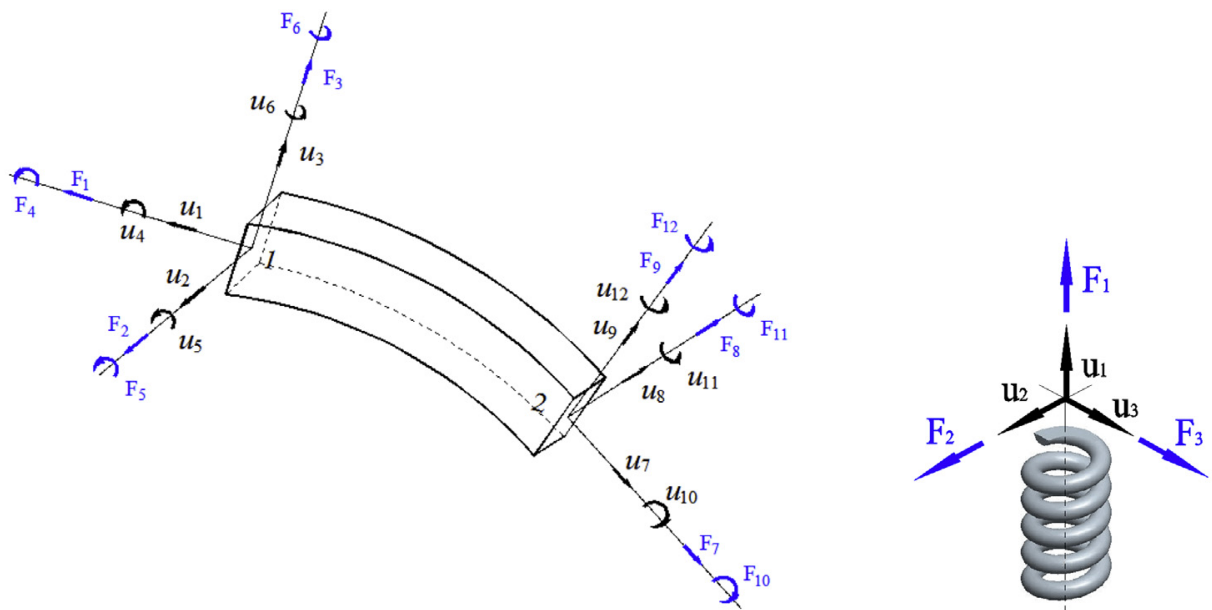

(a) circular-arc beam element (CABE)

(b) extended spring element (ESE)

Fig. 4. Two kinds of one-dimensional elements. 


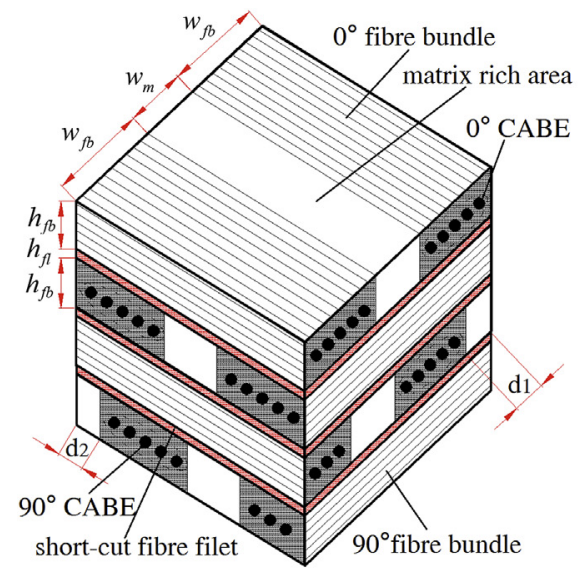

(a) schematic mesoscopic structure

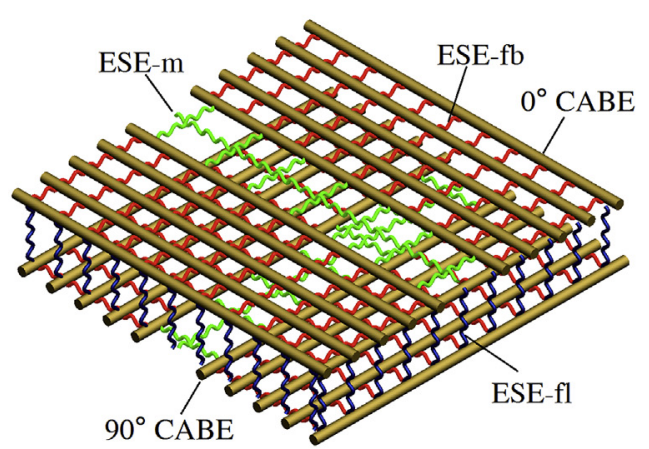

(b) connections of beams and springs

Fig. 5. Beam-spring model of NP-C/C.

\subsection{Equivalent mechanical model of ESE}

3.4.1. Equivalent elasticities of porous pyrocarbon phase and short-cut fibre felt

The pyrocarbon phase in C/Cs usually include a considerable volume percentage of void and this imperfection, inevitable under the recent forming process condition, is non-ignorable in mechanical property analysis. In NP C/Cs, various size, shape and density voids are found in rich matrix area, felt layer, pinhole region likewise matrix phase in fibre bundle. For simplification, void densities are smeared in the matrix phase regions mentioned above, and all the voids are uniformed as spherical shape and supposed as random distribution.

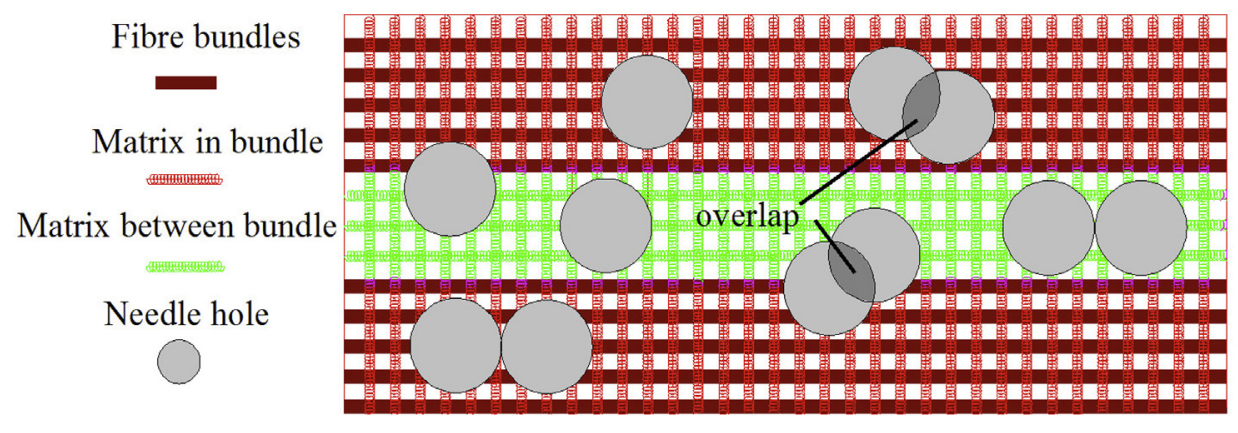

(a) positions of needle holes

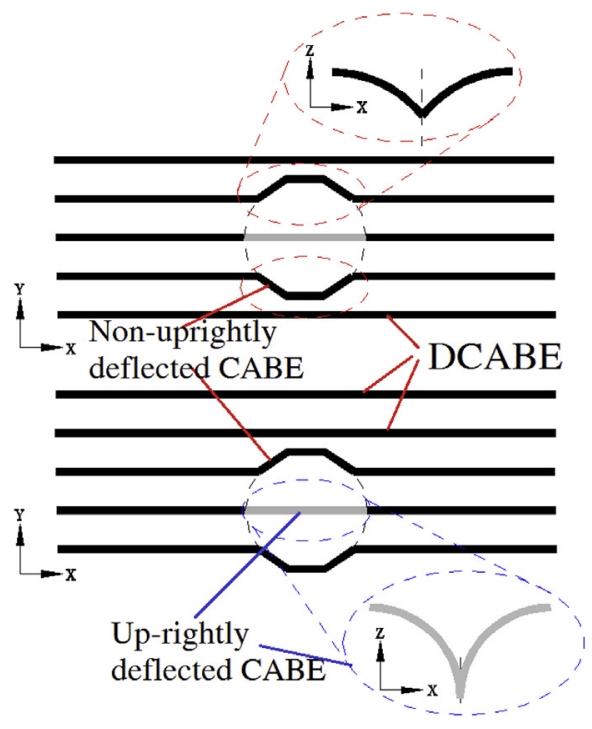

(b) CABEs in punched fibre bundle: Uprightly deflected CABE, Non-uprightly deflected CABE and DCABE

Fig. 6. Mesoscopic defeats model. 


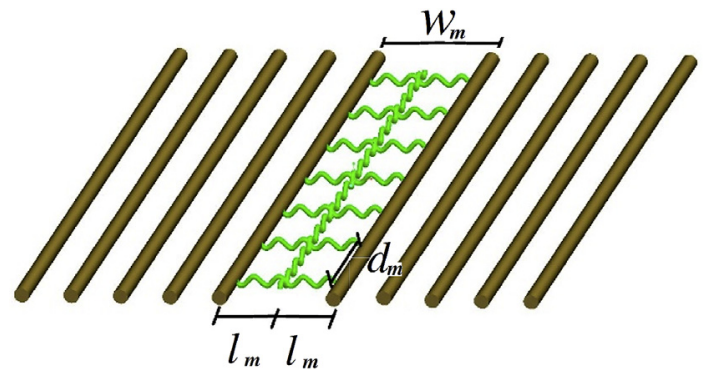

Fig. 7. ESE-ms between fibre bundles in one cloth.

Besides, void size is simplified small enough compared with the media it exists. With all these assumptions, pyrocarbon phase in $\mathrm{C} / \mathrm{Cs}$ is viewed as isotropic porous material, and its elasticity can be estimated by mechanical models for multiphase or porous materials, for example Mori-Tanaka model as we employed in the previous research $[13,14]$, the effective bulk and shear moduli $\left(\kappa_{m}\right.$ and $G_{m}$ ) of porous pyrocarbon phase are expressed [14]:

$\kappa_{m}=\kappa_{0}\left[1+\frac{c_{v}}{\left(1-c_{v}\right) \alpha-1}\right] ; G_{m}=G_{0}\left[1+\frac{c_{v}}{\left(1-c_{v}\right) \beta-1}\right]$

where $\alpha=\frac{1+v_{m}}{3\left(1-v_{m}\right)}, \beta=\frac{8-10 v_{m}}{15\left(1-v_{m}\right)}, \kappa_{0}$ and $G_{0}$ are the bulk and shear moduli of the pure matrix phase, respectively, $c_{v}$ is the volume fraction of voids, $v_{m}$ is the Poisson's ratio of the matrix. So, the effective Young's modulus of porous matrix can be calculated from the effective bulk and shear moduli: $E_{m}=9 G_{m} \kappa_{m} /\left(3 \kappa_{m}+G_{m}\right)$.

The elastic properties of aligned short-cut fibre felt are estimated by Halpin-Tsai equation [15]:

$E_{11}^{f l}=\frac{1+2 \eta \frac{\bar{E}_{11}^{f}-1}{\bar{E}_{11}^{f}+2 \eta} c_{f l}}{1-\frac{\bar{E}_{11}^{f}-1}{\bar{E}_{11}^{f}+2 \eta} c_{f l}} E_{m}, E_{22}^{f l}=\frac{1+\frac{\bar{E}_{22}^{f}-1}{2 \bar{E}_{22}^{f}+1} c_{f l}}{1-\frac{\bar{E}_{22}^{f}-1}{\bar{E}_{22}^{f}+0.5} c_{f l}} E_{m}$

$G_{12}^{f l}=G_{13}^{f l}=\frac{1+\frac{\bar{G}_{12}^{f}-1}{\bar{G}_{12}^{f}+1} c_{f l}}{1-\frac{\bar{G}_{12}^{f}-1}{\bar{G}_{12}^{f}+1} c_{f l}} G_{m}, G_{23}^{f l}=\frac{1+\frac{\bar{G}_{23}^{f}-1}{2 \bar{G}_{23}^{f}+1} c_{f l}}{1-\frac{\bar{G}_{23}^{f}-1}{\bar{G}_{23}^{f}+0.5} c_{f l}} G_{m}$

where, $E_{11}^{f l}$ and $E_{22}^{f l}$ are its longitudinal and transverse moduli, $G_{23}^{f l}$ and $G_{12}^{f l}\left(G_{13}^{f l}\right)$ are its in-plane and out-plane shear moduli. $\bar{E}_{11}^{f}=E_{11}^{f} / E_{m}$ and $\bar{E}_{22}^{f}=E_{22}^{f} / E_{m}$ are the normalized longitudinal and transverse moduli of short fibre, $\bar{G}_{12}^{f}=G_{12}^{f} / G_{m}$ and $\bar{G}_{23}^{f}=G_{23}^{f} / G_{m}$ are the normalized longitudinal and transverse shear moduli of short fibre, respectively. $\eta$ is the aspect ratio of short fibre and $c_{f l}$ is the volume fraction of short fibre in felt.

The felt of randomly oriented short-cut fibre is supposed in-plane isotropic and its in-plane elastic moduli are given by empirical equations [15]:

$E_{i n}^{f l}=\frac{3}{8} E_{11}^{f l}+\frac{5}{8} E_{22}^{f l}$

$G_{i n}^{f l}=\frac{1}{8} E_{11}^{f l}+\frac{1}{4} E_{22}^{f l}$

where, $E_{i n}^{f l}$ and $G_{i n}^{f l}$ are the in-plane Young's modulus and shear modulus of the felt ply, respectively.

Considering the thickness of short-cut felt is much smaller than that of cloth ply, the out-plane elastic modulus of randomly oriented shortcut felts $\left(E_{\text {out }}^{f l}\right)$ is supposed same as $E_{22}^{f l}$ for simplification. While the randomly oriented short-cut felt is viewed composed with some unidirectional short fibre layers and their angles distribute randomly inplane, so the out-plane shear modulus $G_{\text {out }}^{f l}$ can be simplified as average of longitudinal and transverse shear moduli of aligned short fibre layers:

$G_{\text {out }}^{f l}=\frac{1}{2}\left(G_{13}^{f l}+G_{23}^{f l}\right)$

\subsubsection{Equivalent elasticities of ESE-m}

The stiffnesses of ESE-m are calculated according to the dimension of the binding region which substituted by one ESE-m element in the sense of binding stiffness equivalent:

$K_{1}^{m-l}=\frac{E_{m} h_{f b} l_{m}}{d_{m}}, K_{1}^{m-t}=\frac{E_{m} h_{f b} d_{m}}{l_{m}}$

$K_{2}^{m-l}=K_{3}^{m-l}=\frac{G_{m} h_{f b} l_{m}}{d_{m}}, K_{2}^{m-t}=K_{3}^{m-t}=\frac{G_{m} h_{f b} d_{m}}{l_{m}}$

where, $K_{i}^{m-l}$ and $K_{i}^{m-t}$ are stiffness of ESE-ms along and transverse to fibre bundle. The subscript $i=1$ denotes normal direction of spring element while $i=2,3$ denote the two shear directions of it, and this definition is after versa. As demonstrated in Fig. 7, $d_{m}$ and $l_{m}$ are the lengths of ESE-ms along and transverse to fibre bundle.

\subsubsection{Equivalent elasticities of ESE- $f b$}

The equivalent stiffnesses of ESE-fb (Fig. 8 (a), red color ESE) are calculated as:

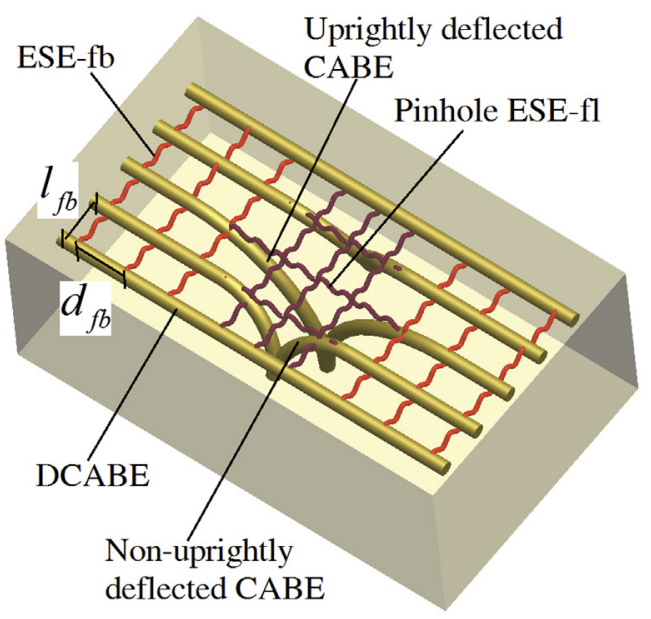

(a) a centrally punched fibre bundle

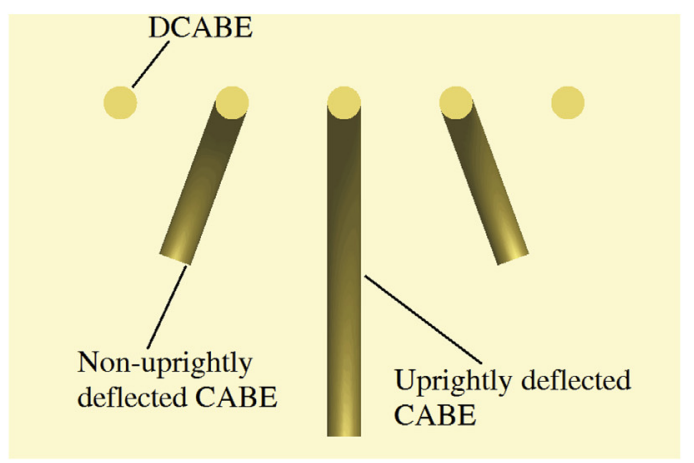

(b) local view of CABEs at punching point

Fig. 8. Beam-spring element model of a fibre bundle. 


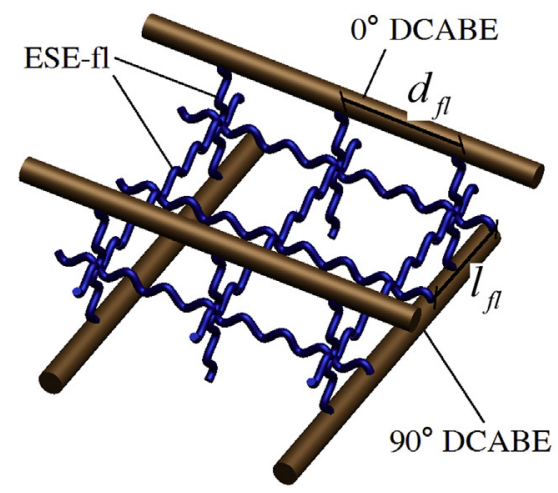

(a) un-punched area

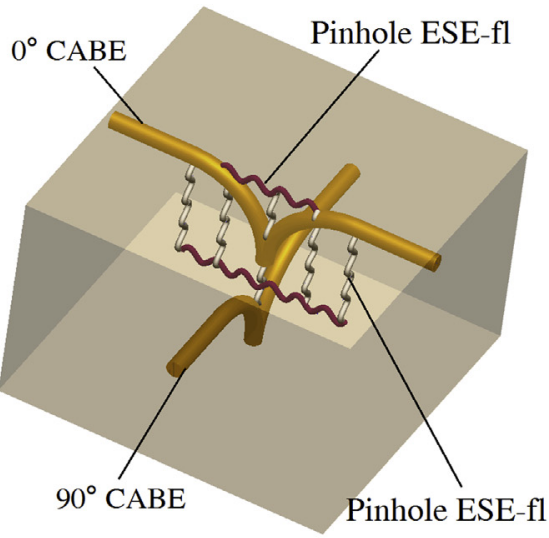

(b) pinhole area

Fig. 9. ESE-fls in un-punched and punched areas.

Table 1

Mesoscopic structure parameters of NP C/Cs (unit: mm).

\begin{tabular}{llcc}
\hline$h_{f b}$ & $h_{f l}$ & $w_{f b}$ & $w_{m}$ \\
\hline 0.42 & 0.08 & 1.50 & 0.90 \\
\hline
\end{tabular}

Table 2

Dimension parameters of beam and spring elements (unit: $\mathrm{mm}$ ).

\begin{tabular}{llllll}
\hline$l_{m}$ & $d_{m}$ & $l_{f b}$ & $d_{f b}$ & $l_{f l}$ & $d_{f l}$ \\
\hline 0.30 & 0.30 & 0.30 & 0.30 & 0.30 & 0.30 \\
\hline
\end{tabular}

Table 3

Mechanical parameters of carbon matrix.

\begin{tabular}{llll}
\hline$E_{m} / \mathrm{GPa}$ & $G_{m} / \mathrm{GPa}$ & $X_{m} / \mathrm{MPa}$ & $S_{m} / \mathrm{MPa}$ \\
\hline 8.46 & 3.38 & 12.30 & 16.00 \\
\hline
\end{tabular}

$K_{1}^{f b}=\frac{E_{f b}^{t} h_{f b} d_{f b}}{l_{f b}}$

$K_{2}^{f b}=\frac{G_{f b}^{l} h_{f b} d_{f b}}{l_{f b}}, K_{3}^{f b}=\frac{G_{f b}^{t} h_{f b} d_{f b}}{l_{f b}}$

where, $E_{f b}^{t}$ is the transverse modulus of the fibre bundle, $G_{f b}^{l}$ and $G_{f b}^{t}$ are the longitudinal and transverse shear moduli of the fibre bundle, respectively. $l_{f b}$ and $d_{f b}$ are the length of a ESE-fb and the interval of two adjacent ESE-fbs. Fig. 8(b) is the local view of CABEs' deflections at punching point.

\subsubsection{Equivalent elasticities of ESE-fl}

The equivalent stiffness of intra-laminar ESE-fl (Fig. 9 (a)) are calculated as:

$K_{1}^{f l-l}=\frac{E_{i n}^{f l} h_{f l} l_{f l}}{d_{f l}}, K_{1}^{f l-t}=\frac{E_{i n}^{f l} h_{f l} d_{f l}}{l_{f l}}$
Table 5

Mechanical parameters of short-cut carbon fibre and felts.

\begin{tabular}{llllllll}
\hline$E_{\text {in }}^{\text {fl }} / \mathrm{GPa}$ & $E_{\text {out }}^{\text {fl }} / \mathrm{GPa}$ & $G_{\text {in }}^{\text {fl }} / \mathrm{GPa}$ & $G_{\text {out }}^{\text {fl }} / \mathrm{GPa}$ & $\bar{E}_{11}^{f}$ & $\bar{E}_{22}^{f}$ & $c_{f l} / \%$ & $\eta$ \\
\hline 17.36 & 11.79 & 6.18 & 4.64 & 27.19 & 4.73 & 10.00 & 8.57 \\
\hline
\end{tabular}

$K_{2}^{f l-l}=\frac{G_{i n}^{f l} h_{f l} l_{f l}}{d_{f l}}, K_{2}^{f l-t}=\frac{G_{i n}^{f l} h_{f l} d_{f l}}{l_{f l}}$

$K_{3}^{f l-l}=\frac{G_{\text {out }}^{f l} h_{f l} l_{f l}}{d_{f l}}, K_{3}^{f l-t}=\frac{G_{\text {out }}^{f l} h_{f l} d_{f l}}{l_{f l}}$

Where $K_{i}^{f l-l}$ and $K_{i}^{f l-t}$ are stiffnesses of ESE-fls along $0^{\circ}$ and $90^{\circ}$ fibre bundles, $l_{f l}$ and $d_{f l}$ are the lengths of these two ESE-fls. The equivalent stiffnesses of inter-laminar ESE-fl are calculated as:

$K_{1}^{f l-v}=\frac{E_{\text {out }}^{f l} d_{f l} l_{f l}}{h_{f l}}$

$K_{2}^{f l-v}=K_{3}^{f l-v}=\frac{G_{\text {out }}^{f l} d_{f l} l_{f l}}{h_{f l}}$

Punching hole is filled with some short carbon fibres brought by the barbed needle from felt ply in punching processes, for simplification these short fibres are assumed vertically aligned in pinhole and the mechanical properties there are represented by vertical and planar spring elements with elasticities of aligned short cut fibre felt, as illustrated in Fig. 9(b). These spring elements are denoted as pinhole ESEfls, and the equivalent stiffnesses of vertical pinhole ESE-fl (silver springs in Fig. 9 (b)) are estimated as:

$K_{1 P}^{f l-v}=\frac{E_{11}^{f l} d_{f l}^{\prime} l_{f l}^{\prime}}{h_{f l}}$

$K_{2 P}^{f l-v}=K_{3 P}^{f l-v}=\frac{G_{i n}^{f l} d_{f l}^{\prime} l_{f l}^{\prime}}{h_{f l}}$

The equivalent stiffnesses of pinhole ESE-fls in two intra directions (crimson springs in Figs. 8(a) and 9 (b)) are:

Table 4

Mechanical parameters of fibre bundle.

\begin{tabular}{|c|c|c|c|c|c|c|c|c|}
\hline$E_{f b}^{l} / \mathrm{GPa}$ & $E_{f b}^{t} / \mathrm{GPa}$ & $G_{f b}^{l} / \mathrm{GPa}$ & $G_{f b}^{t} / \mathrm{GPa}$ & $X_{f b}^{t} / \mathrm{MPa}$ & $X_{f b}^{c} / \mathrm{MPa}$ & $Y_{f b}^{t} / \mathrm{MPa}$ & $Y_{f b}^{c} / \mathrm{MPa}$ & $S_{f b}^{l} / \mathrm{MPa}$ \\
\hline 86.40 & 10.70 & 6.02 & 5.04 & 927.50 & 463.75 & 11.10 & 51.40 & 13.30 \\
\hline
\end{tabular}




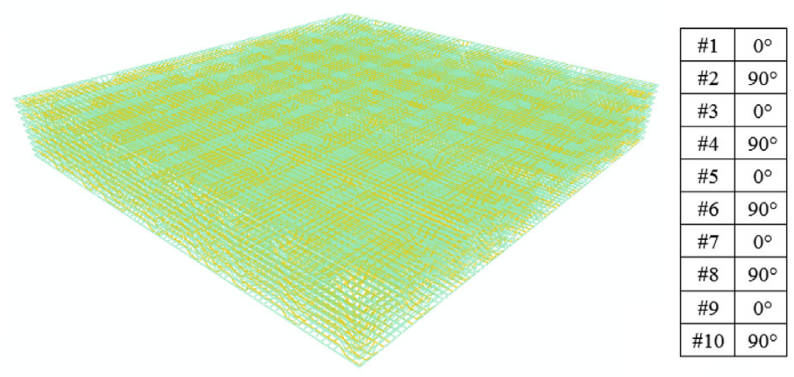

(a) global view

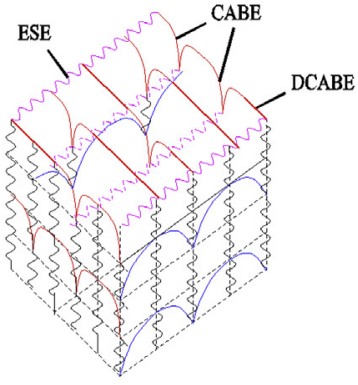

(b) local view

Fig. 10. Mesoscopic numerical model of NP C/Cs with CABE (DCABE) and ESEs.

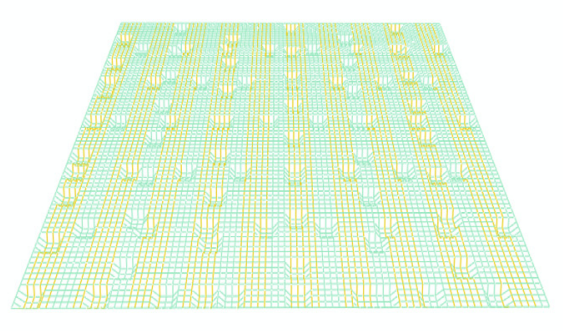

(a) $0^{\circ}$ UD ply

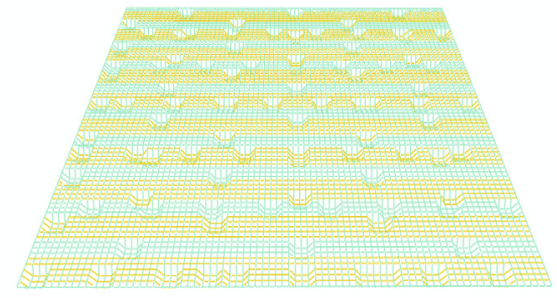

(b) $90^{\circ} \mathrm{UD}$ ply

Fig. 11. Distributions of needle holes in UD plies.

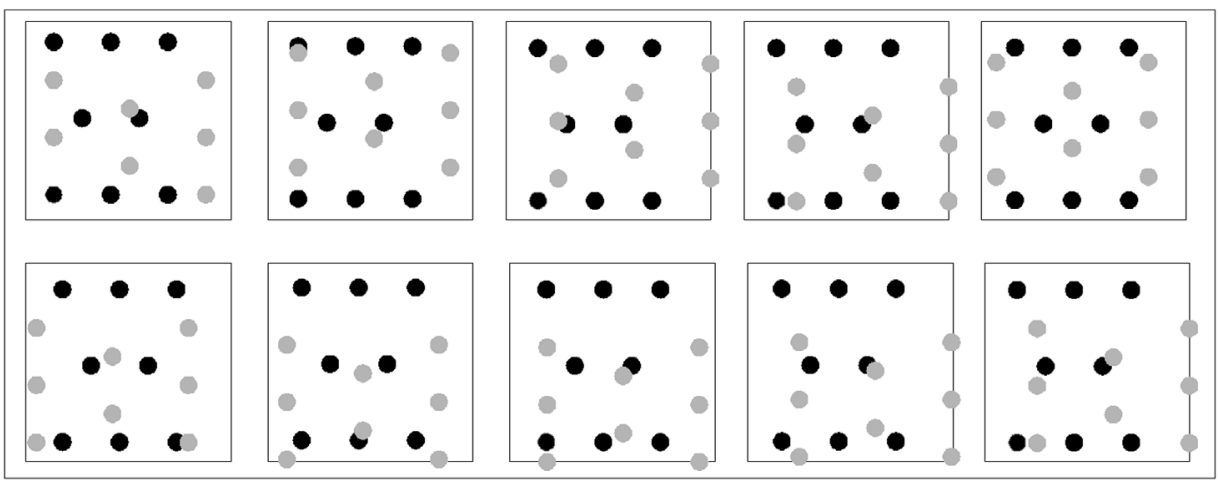

Fig. 12. Random needle holes' positions by twice punching.

$K_{1 P}^{f l-l}=\frac{E_{22}^{f l} h_{f l} l_{f l}^{\prime}}{d_{f l}^{\prime}}, K_{1 P}^{f l-t}=\frac{E_{22}^{f l} h_{f l} d_{f l}^{\prime}}{l_{f l}^{\prime}}$

$K_{2 P}^{f l-l}=K_{3 P}^{f l-l}=\frac{G_{\text {out }}^{f l} h_{f l} l_{f l}^{\prime}}{d_{f l}^{\prime}}, K_{2 P}^{f l-t}=K_{3 P}^{f l-t}=\frac{G_{\text {out }}^{f l} h_{f l} d_{f l}^{\prime}}{l_{f l}^{\prime}}$

In pinhole region the lengths of $d_{f l}$ and $l_{f l}$ need to be modified according to the shapes of needle holes, and the modified ones named $d_{f l}^{\prime}$ and $l_{f l}^{\prime}$ respectively.

Longitudinal maximum stress failure of CABE and quasi-brittle failure of matrix-rich area are employed from previous work [13,16-18].

\section{Simulation and discussions}

\subsection{Numerical RVE modelling}

A series of numerical RVE models composed with CABEs and ESEs are built for investigating the dependencies of mechanical behaviors of $\mathrm{NP} \mathrm{C} / \mathrm{C}$ on its meso-scale featured parameters. According to the parameters of single ply and measurement, the mesoscopic model dimensions, as denoted in Figs. 5 and 7-9, are determined and listed in Tables 1 and 2.

In these models, each UD and felt plies are discretized to a square grid mesh and they are connected by ESEs in thickness. In UD ply each fibre bundle is composed with five rows of CABEs and (or) DCABEs. The dimensions of all elements are listed in Table 2. Mechanical properties of the carbon matrix and the fibre bundles are given in Tables 3 and 4, they were obtained in our previous work [13]. Mechanical properties of carbon felts are predicted by Eq. (3) (6) and the results are shown in Table 5. In pinhole regions, the positions of nodes, together with the lengths of relevant elements should be modified according to the shapes of needle holes, while the number and connection topology of elements are invariant. All the numerical RVE models have dimension of $24 \mathrm{~mm} \times 24 \mathrm{~mm} \times 5 \mathrm{~mm}$ and are discretized to $0.3 \mathrm{~mm} \times 0.3 \mathrm{~mm}$ square mesh in plane and $0.25 \mathrm{~mm} \times 0.3 \mathrm{~mm}$ rectangular mesh on lateral sides. There are totally 39500 CABEs (DCABEs) and 144500 ESEs in a model. Fig. 10 gives the global and local views of a numerical RVE model in which fibre bundles in the same direction are aligned perfectly along thickness. The cloth plies numbered from \#1 to \#10 are 


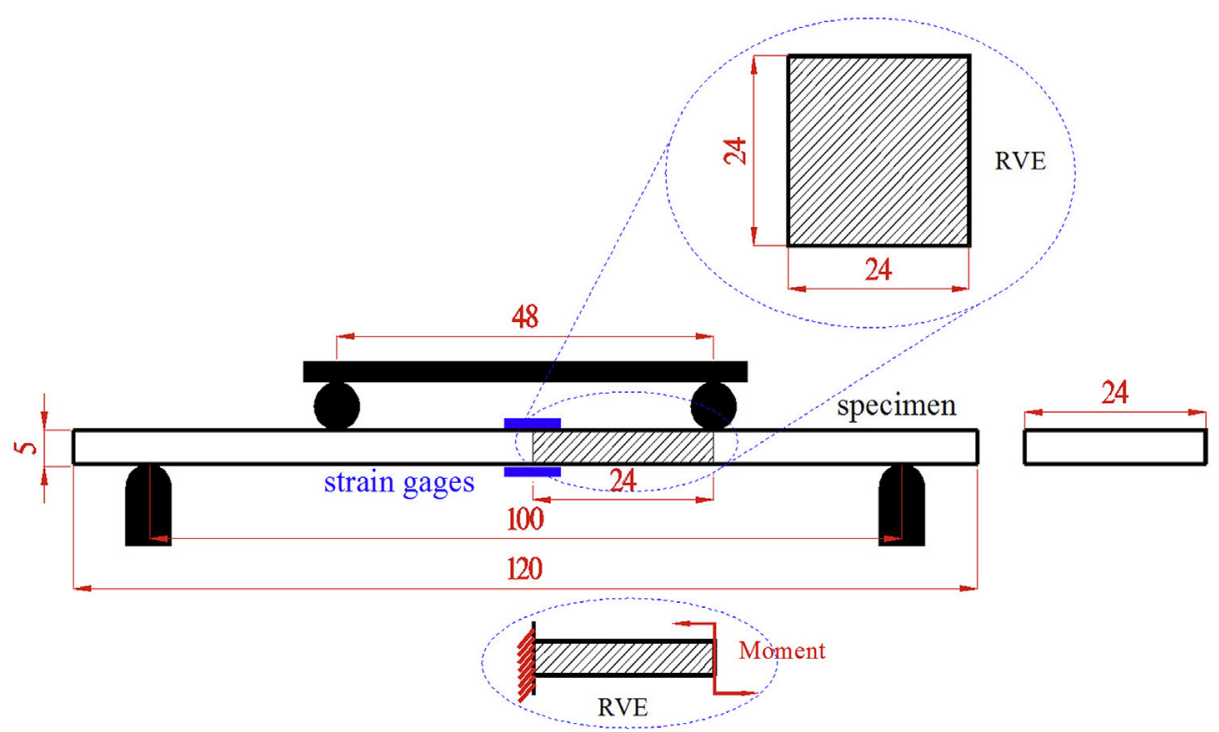

Fig. 13. Four-point bending test and boundary condition of its simulation model.

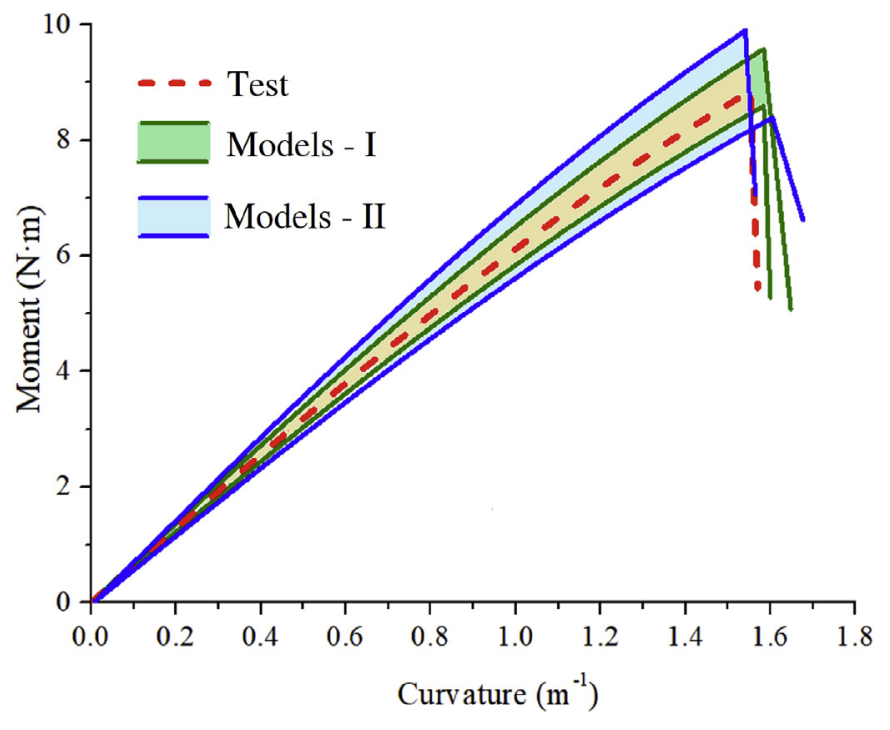

Fig. 14. Experimental and predicted moment-curvature curves for NP C/C.

counted from top surface to bottom surface. The punched needle holes distributions in UD cloth plies are shown in Fig. 11. The cyan color lines in Figs. 10 and 11 represent CABEs (and DCABEs) while the claybank color lines represent ESEs.

Random of mesoscale structure (including punching introduced defects) is the inherent feature of NP C/Cs $[19,20]$ and its influence on the dispersion of mechanical properties of this composite is intent to investigate in this study. Two methods are used to generate random mesoscale structure in numerical RVE models here:

(1) In RVE the position of one $0^{\circ} \mathrm{UD}$ ply is fixed while the other $0^{\circ}$ plies are plied with random offsets to the fixed ply, in the other words $d_{1}$ in Fig. 5(a) is set a random value within scope from zero to value of $w_{f b}+w_{m}$. The $90^{\circ}$ plies are stacked in the same way. This type models are named Models-I in this paper, and for them the positions of needle holes are identical as shown in Fig. 3(c).

(2) The relative positions of first punching and second punching are set in random, while all the $0^{\circ}$ and $90^{\circ}$ plies are set aligned perfectly along thickness. The actual practice is that the coordinates of first punching needle holes (black solid circles in Fig. 12) are set fixed in RVE for all the models, and the coordinates of second punching needle holes (grey solid circles in Fig. 12) are set by random data generated by computer. The random data used should let all the second time needle holes locate inside the RVE scope in order that all the RVE models have the same punching density. The needle holes' positions of ten numerical RVE models (named Models-II) generated by this method are illustrated in Fig. 12.

\subsection{Four-point experiment}

The developed numerical models were employed to simulate quasistatic four-point-bending experiments, as schematically illustrated in Fig. 13. The dimensions of bending-test specimens were $120 \mathrm{~mm} \times 24 \mathrm{~mm} \times 5 \mathrm{~mm}$, its effective span was $48 \mathrm{~mm}$ in pure bending region and contained two RVEs. Two strain gages were arranged on the top and bottom surfaces on specimen at central section. The experiments were carried out with the universal testing machine CMT5504. The loading rate was controlled at $0.5 \mathrm{~mm} / \mathrm{min}$ and the tensile and compressive strains at the two surfaces were recorded at frequency of 10 times per second. The experimental average curves of moment versus the curvature are shown in Fig. 14 (dash lines). The curvature of specimen at central section is obtained from the equation $\rho_{\text {test }}=\left(\left|\varepsilon_{1}-\varepsilon_{2}\right|\right) / h$, where $\varepsilon_{1}$ and $\varepsilon_{2}$ are values of two strain gages, respectively, $h$ is thickness of specimen.

Table 6

Statistical parameters of predicted mechanical properties of NP C/Cs.

\begin{tabular}{|c|c|c|c|c|c|c|c|c|}
\hline & $E_{\min } / \mathrm{GPa}$ & $E_{\max } / \mathrm{GPa}$ & $E_{\text {ave }} / \mathrm{GPa}$ & $S_{E}$ & $\sigma_{\min } / \mathrm{MPa}$ & $\sigma_{\max } / \mathrm{MPa}$ & $\sigma_{\text {ave }} / \mathrm{MPa}$ & $S_{\sigma}$ \\
\hline Experiment & 25.03 & 25.69 & 25.36 & 0.33 & 87.06 & 89.88 & 88.47 & 1.41 \\
\hline Models-I & 24 & 27 & 25.48 & 0.72 & 86 & 96 & 91.97 & 2.41 \\
\hline Models-II & 23 & 28 & 25.32 & 1.17 & 84 & 99 & 91.67 & 3.56 \\
\hline
\end{tabular}




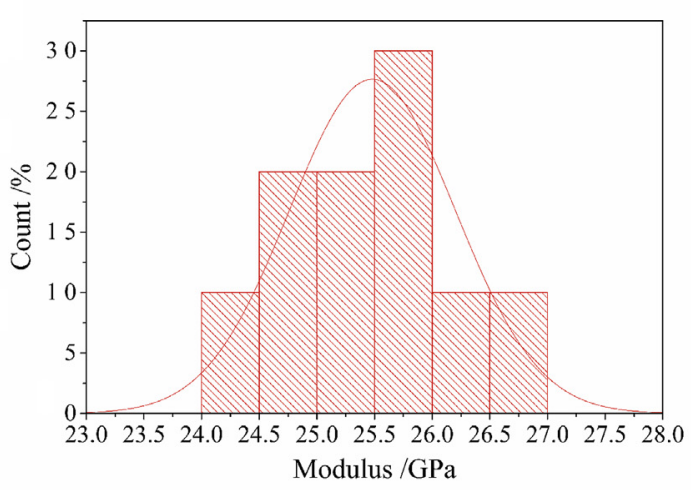

(a) bending modulus

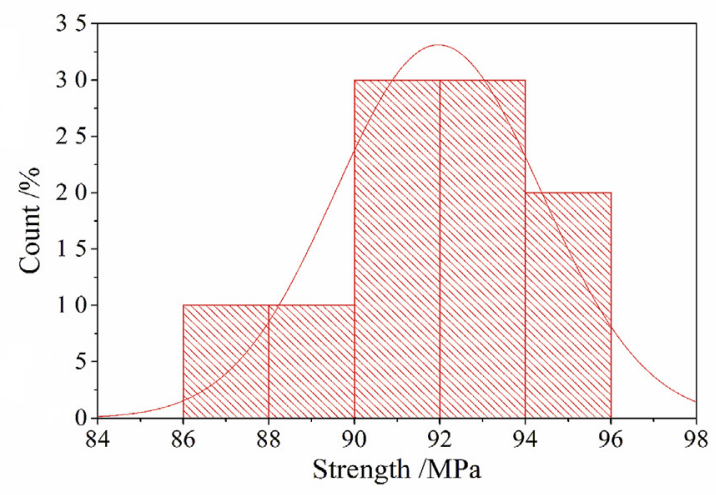

(b) bending strength

Fig. 15. Statistical distributions of bending properties of models-I.

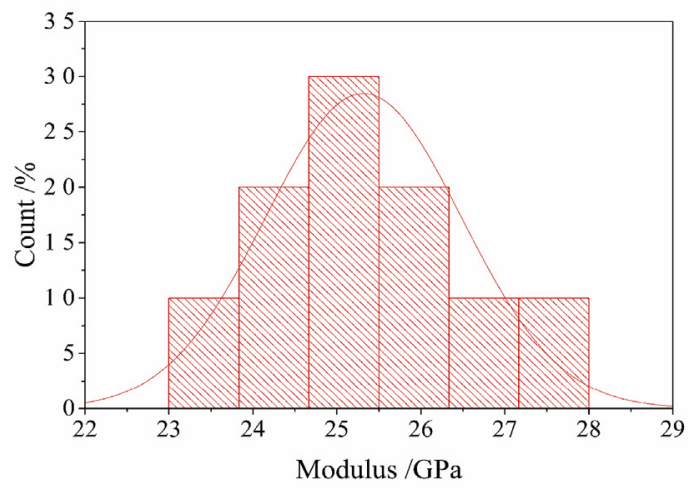

(a) bending modulus

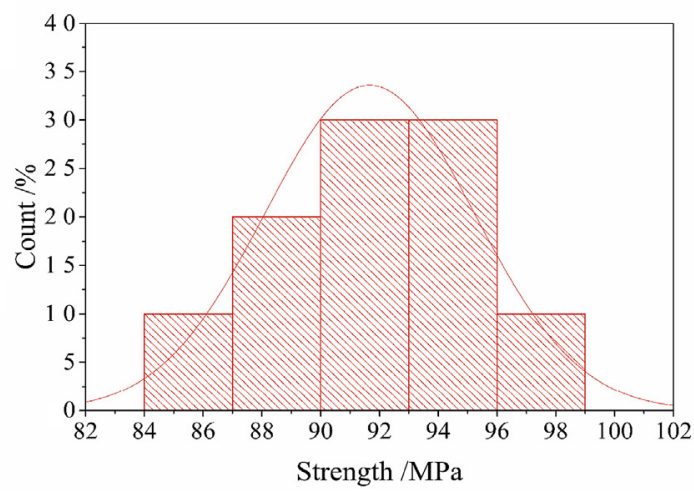

(b) bending strength

Fig. 16. Statistical distributions of bending properties of models-II.

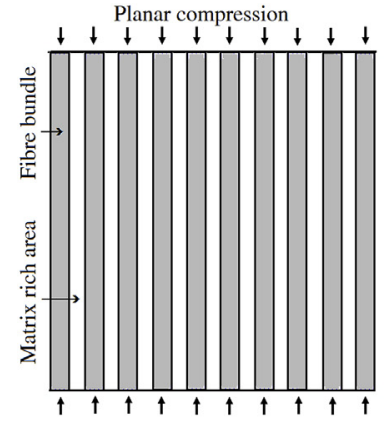

(a) planar compression

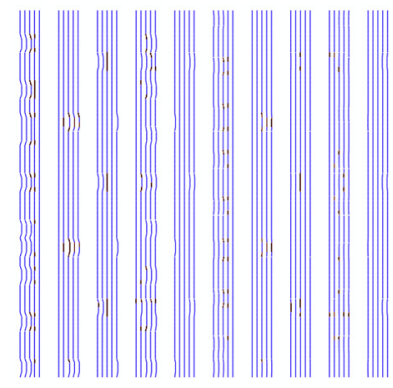

(d) $\mathrm{CABE}$ damage $=0.85$

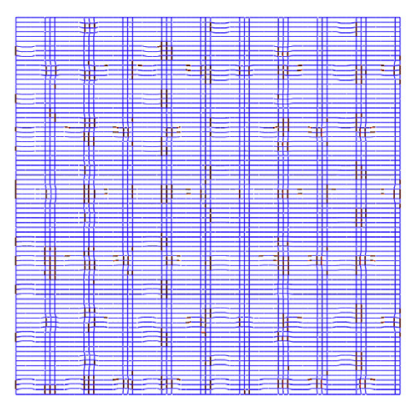

(b) ESEs damage

$=0.6$

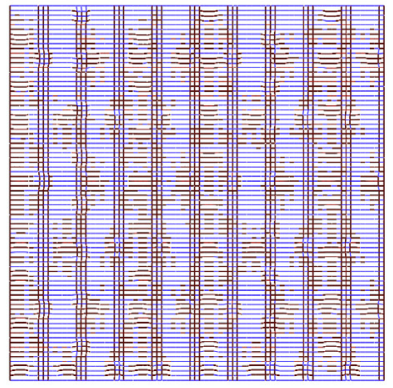

(e) ESEs damage $=1.6$

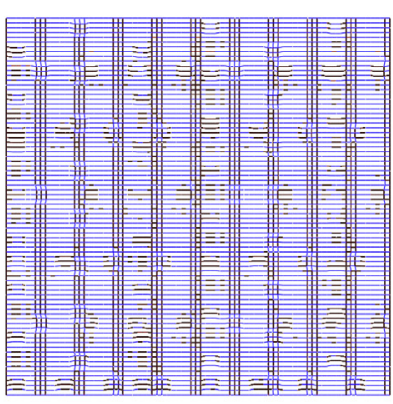

(c) ESEs damage $\quad=0.85$

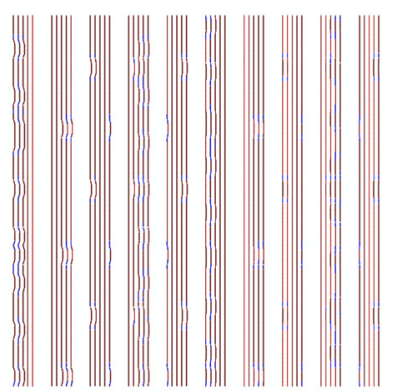

(f) $\mathrm{CABE}$ damage $=1.6$

Fig. 17. Progressive damages in Layer \#1 ( $\left.0^{\circ} \mathrm{UD}\right)$. 

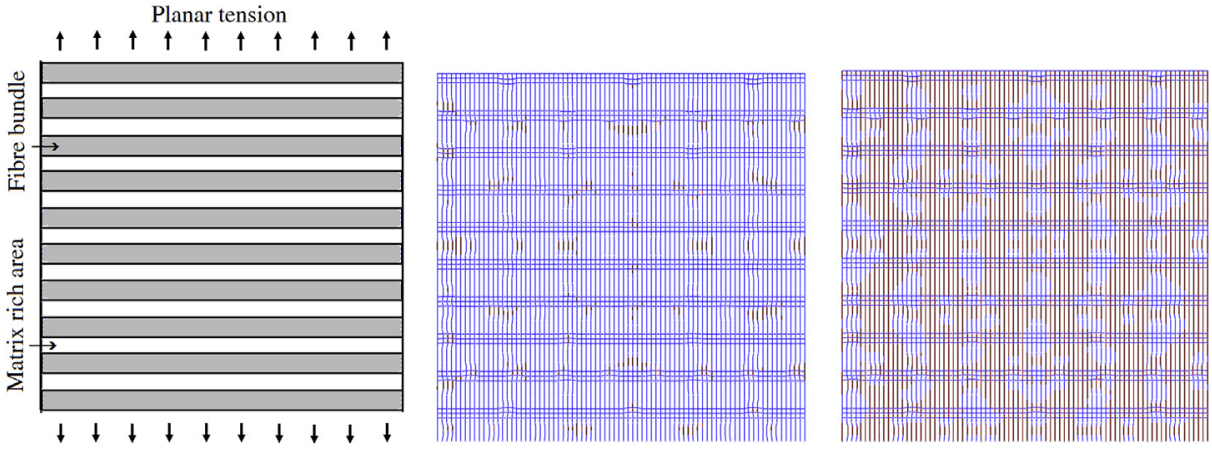

(a) planar tension

(b) ESEs damage $\quad=0.2$

(c) ESEs damage $\quad=0.85$

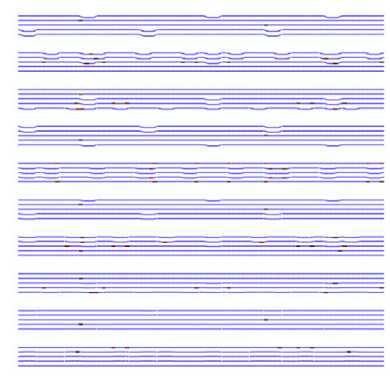

(d) CABE damage

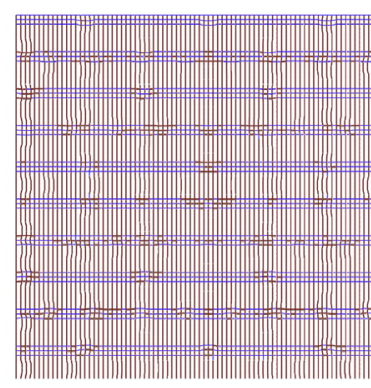

(e) ESEs damage $\quad=1.6$

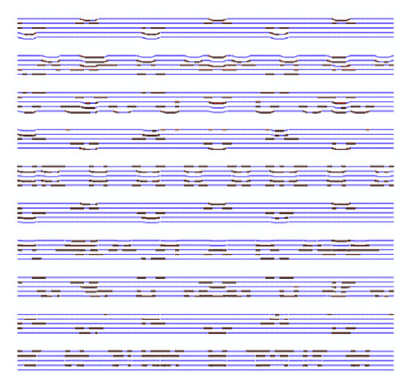

(f) $\mathrm{CABE}$ damage $=1.6$

Fig. 18. Progressive damages in Layer $\# 10$ ( $90^{\circ}$ UD).

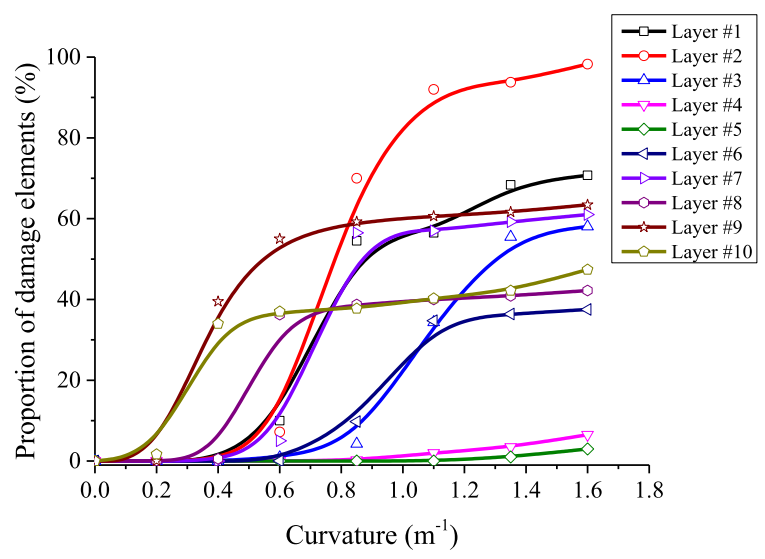

(a) ESE-m

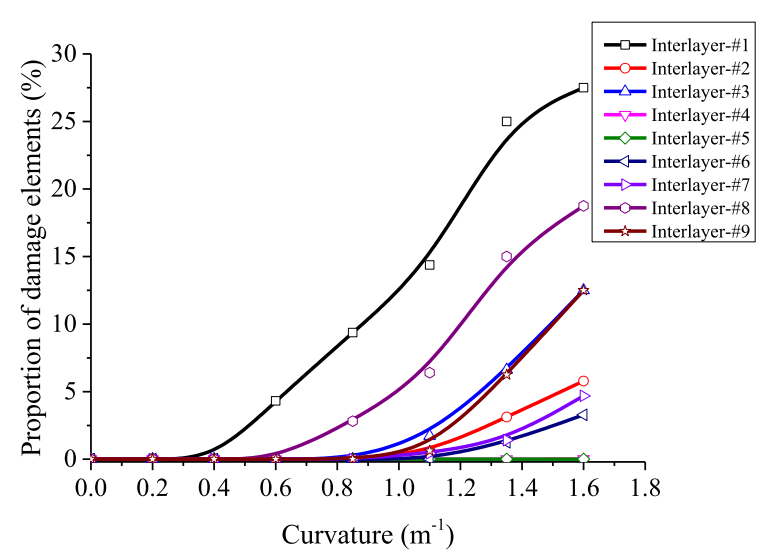

(c) ESE-fl

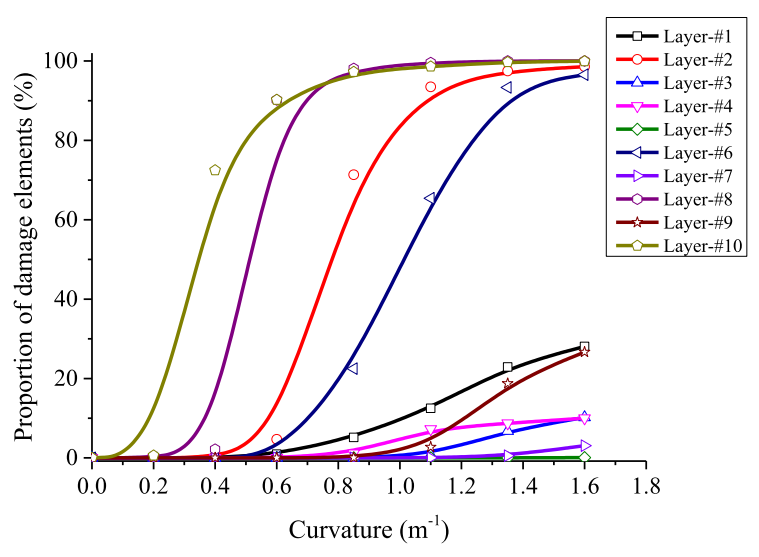

(b) ESE-fb

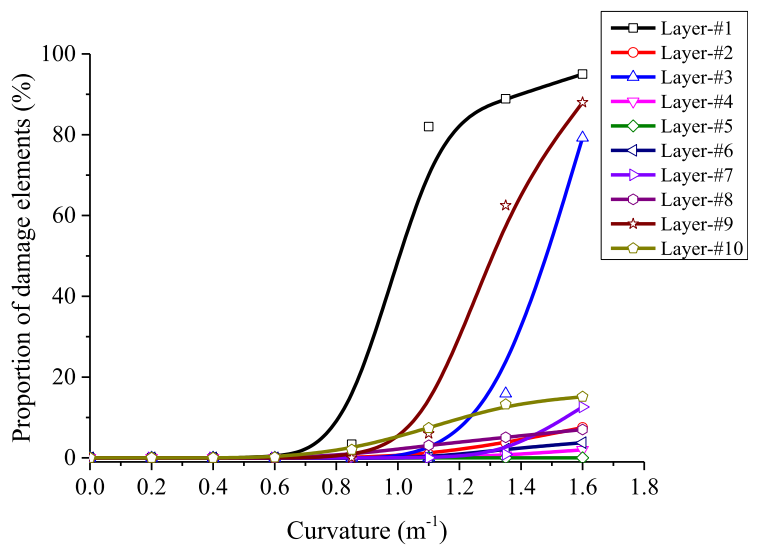

(d) $\mathrm{CABE}$ (or DCABE)

Fig. 19. Evolutions of multi damages in various plies. 


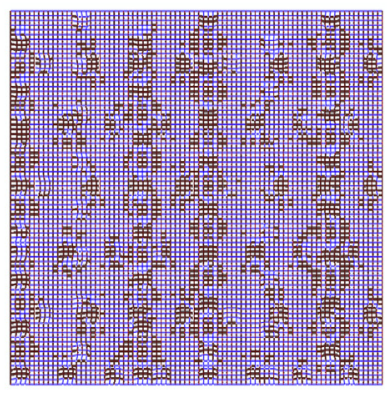

(a) layer \#1

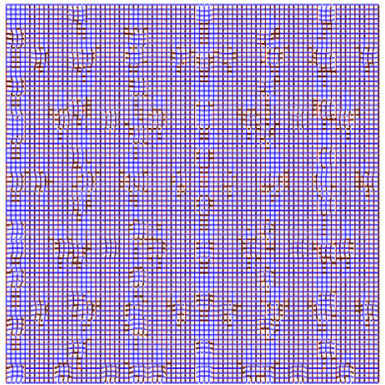

(d) layer \#9

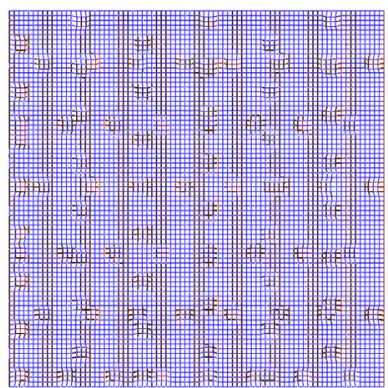

(b) layer \#3

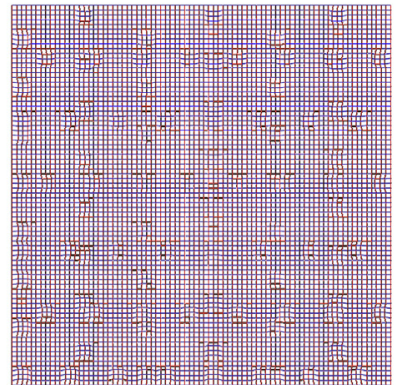

(e) layer \#8

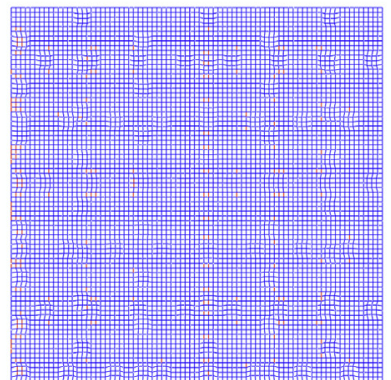

(c) layer \#5

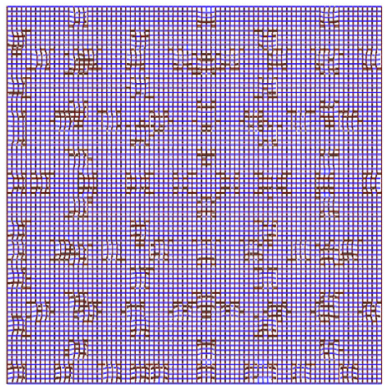

(f) layer \#10

Fig. 20. Damage distributions in various plies under failure bending load.

\subsection{Numerical simulation and results discussion}

As schematically shown in Fig. 13, only half of the pure bending section in specimen is used in numerical simulation considering symmetry condition, and the dimension of this section is just the same with the RVE. The lateral side of RVE model corresponding to the central cross section of specimen is clamped, its opposite side is applied pure bending load through rigid rotation, while the two other lateral sides and upper and bottom surfaces are free of any constraint. Simulations are conducted on the two sorts of RVE models and the average curvature of RVE is estimated as $\rho_{F E M}=\alpha / L$, where $\alpha$ is rotation displacement of loading point and $L$ is the length of RVE. Curvature in pure bending section of specimen is considered identical. The predicted bending moduli, strengths and their deviations are listed in Table 6, where $S_{E}$ and $S_{\sigma}$ are the standard deviation of bending modulus and bending strength, respectively. The upper and lower bounds of predicted moment-curvature curves are also presented in Fig. 14. The average experimental curve lies between these bounds. Discrepancies for both bending moduli and bending strengths predicted by these two random patterns are found obvious. The dispersion band of momentcurvature curve for the models-II is wider than that for models-I, and the largest dispersion does not over $20 \%$. The statistical predicted bending properties can be approximately described by normal distributions as illustrated in Fig. 15 and Fig. 16.

Fig. 17 shows evolutions of multi damages in layer \#1, the outmost $0^{\circ}$ ply which is under planar compression along fibre bundle direction for pure bending load as shown in Fig. 17(a). The blue segments in the picture denote intact elements and the red ones denote failure elements and this denotation is after versa. The damage firstly appears in ESE-m and ESE-fb which inside or around the needle holes, when curvature $\rho$ reaches 0.6 (planar compressive strain in this layer is $-1550 \mu \varepsilon$ accordingly) $10 \%$ of ESE-m and $0.96 \%$ of ESE-fb have damaged (Fig. 17 (b)). When $\rho$ increases to 0.85 (strain of $-2200 \mu \varepsilon$ accordingly) ESEs failure region further extends (Fig. 17(c)) and CABEs in or around punching holes begin to fail (Fig. 17 (d)). Under final failure bending load of specimen ( $\rho=1.6$, strain of $-3900 \mu \varepsilon$ accordingly) $70.75 \%$ of ESE-m, $28.08 \%$ of ESE-fb and almost $90 \%$ of CABEs are broken, as pictured in Fig. 17(e) and (f).

For layer \#10, the outmost $90^{\circ}$ ply under planar transverse tension as schematically shown in Fig. 18(a), damage initiates in matrix phase in fiber bundle (ESE-fb) inside or around the needle holes. Its failure proportion is $0.625 \%$ when curvature is 0.2 (planar tension strain is $600 \mu \varepsilon$ accordingly), as shown in Fig. 18(b). When curvature increases to 0.85, most ESE-m and ESE-fb have failed (Fig. 18(c)) and transverse damages begin to appear in CABEs inside or around needle holes (Fig. 18(d)). At critical curvature of 1.6, 47.38\% of ESE-m and almost all of ESE-fb have failed in this layer (Fig. 18(e)) while only $15.15 \%$ of CABEs damage transversely (Fig. 18(f)) and of them still locate inside or around of the needle holes.

The damage developments of various phases (or elements) in RVE are presented in Fig. 19. The matrix damages appear earlier than in felt and fiber bundle phases and they trend to approach saturate with curvature increasing. The matrix damages in $90^{\circ}$ layers (even number layers) more severely than in $0^{\circ}$ layers (odd number layers) because in the latter most loads are carried by fibre. Felt phase damage appears later than matrix phases because the short fibre in it can effectively strengthen its strength and toughness, damages of felt layers keep increasing during the whole loading process. Fiber damage, i.e. damage of CABE (or DCABEs), happens latest among all the damages. From Fig. 19(d), one can find CABE damages in outside $0^{\circ}$ layers (layers \#1, \#3 and \#9) are much more severe than in other layers, and this implies that most bending loads are burdened by the outside $0^{\circ}$ layers. Compressive strength of this NP C/C is found lower than its tensile strength in our previous work [13], so CABE damage in layer \#7 is milder than in layer \#3, its approximate symmetrical ply. When rate of CABE failure in top $0^{\circ}$ layer (layer \#1) is approaching $100 \%$, the composite will fail completely. In other words, the bending strength of NP C/C is dominated by the compressive strength of outer $0^{\circ}$ layers. The severities of all damages decrease from surface layers to neutral layers and this trend agrees with the load carrying distribution in plies along thickness direction.

The distributions of damages in some layers under failure bending load are presented in Fig. 20. Layer \#1 and layer \#9 locate in outermost, so they have larger damage areas than layer \#3 and layer 5\# which locate in inner region, as pictured in Fig. 20(a) (d). Layer \#5 is the one nearest to neutral plane, so its damage degree is the minimal. Damage area in layer \#1 is larger than in layer \#9 and this attributes to the fact that compressive strength is smaller than tensile strength for 
this composite. Additionally, layer \#1 is slightly farther away from neutral plane than layer \#9. For the same reason $90^{\circ}$ plies exhibit similar damage feature, for example layer \#10 has larger damage area than layer \#8, as illustrated in Fig. 20(e) and (f).

\section{Conclusions}

Micromechanical models which constructed with circle-arc beam elements and extended spring elements are built, and to them two sets of random microstructural parameters, i.e. relative offsets between stacked UD cloth plies (Models-I) and distribution patterns of needle holes (Models-II), are introduced. These models are used for revealing the impacts from inherent randomness of microstructure for this composite to its dispersions of mechanical properties.

Numerical results show that the gaps between predicted upper and lower bounds for both bending modulus and bending strength caused by these two sets of random parameters are obvious, the maximum dispersions of bending stiffness is $11.83 \%$ and of bending strength is $11.30 \%$ for Models-I, and they are $19.72 \%$ and $16.95 \%$ respectively for Models-II. This means a mechanical model with definite microparameters cannot comprehensively reflect mechanical behaviors of NP $\mathrm{C} / \mathrm{Cs}$. The average experimental moment-curvature curve locate between the simulated upper and lower bounds, therefore the validation of the proposed micromechanical models which including random parameter has been proven.

Numerical simulations also find that damage-initiation regions are localized around needle holes and extend along the maximum stress direction, this phenomenon is an evidence that punching process is detrimental for intra-laminate mechanical properties in some levels. Damage firstly appears in matrix phase, especially in matrix of fibre bundle, and this damage approaches saturate with bending load increasing, felt layer damage happens consequently and fibre axial failure appears latest. The compressive load bearing capacity of the outmost $0^{\circ}$ layer dominates the ultimate bending strength of NP C/Cs.

\section{Acknowledgements}

This work is partially supported by the National Natural Science Foundation of China (11872205, 11272147), SKL Open Fund (MCMS0218G01), Foundation of National Scholarship of China (201706830073) and Priority Academic Program Development of Jiangsu Higher Education Institutions.

\section{References}

[1] X.M. Chen, L. Chen, C.Y. Zhang, L.L. Song, D.T. Zhang, Three-dimensional needle- punching for composites- A review, Composites Part A 85 (2016) 12-30.

[2] D.S. Li, G. Luo, Q.Q. Yao, N. Jiang, L. Jiang, High temperature compression properties and failure mechanism of 3D needle-punched carbon/carbon composites, Mater. Sci. Eng. A 621 (2015) 105-110.

[3] D.S. Li, H.W. Duan, W. Wang, D.Y. Ge, L. Jiang, Q.Q. Yao, Strain rate and temperature effect on mechanical properties and failure of 3D needle-punched Carbon/ Carbon composites under dynamic loading, Compos. Struct. 172 (2017) 229-241.

[4] X. Li, S. Yu, Y.P. Li, Q. Wu, Z. Li, T. Xiao, L.H. Liu, X.N. Guo, Effect of pre-fatigue on bending behaviour of 2.5 D C/C composites, Mater. Sci. Eng., A 682 (2017) 290-295.

[5] D.S. Li, Q.Q. Yao, N. Jiang, L. Jiang, Bend properties and failure mechanism of a carbon/carbon composite with a 3D needle-punched preform at room and high temperatures, N. Carbon Mater. 31 (2016) 437-444.

[6] N. Jiang, D.S. Li, Q.Q. Yao, L. Jiang, Influence of temperature on the bending properties and failure mechanism of 3D needle-punched carbon/epoxy composites, Fibers Polym. 18 (2017) 313-321.

[7] M.Y. Zhang, Z.A. Su, J.L. Li, Q.Z. Huang, Bending properties and fracture mechanism of C/C composites with high density preform, Trans. Nonferrous Metals Soc. China 21 (2011) 1795-1800.

[8] J.B. Xie, G.D. Fang, Z. Chen, J. Liang, Numerical and experimental studies on scattered mechanical properties for 3D needled C/C-SiC composites, Compos. Struct. 192 (2018) 545-554.

[9] J.B. Xie, J. Liang, G.D. Fang, Z. Chen, Effect of needling parameters on the effective properties of 3D needled C/C-SiC composites, Compos. Sci. Technol. 117 (2015) 69-77.

[10] H.J. Zhang, C.W. Zhou, Tensile strength prediction of needle-punched carbon/ carbon composites, J. Reinf. Plast. Compos. 35 (2016) 1490-1512.

[11] J. Yu, C.W. Zhou, H.J. Zhang, A micro-image based reconstructed finite element model of needle-punched C/C composite, Compos. Sci. Technol. 153 (2017) 48-61.

[12] Y.Z. Jia, D.M. Liao, H. Cui, A. Ji, X. Bai, M. Yasir, Modelling the needling effect on the stress concentrations of laminated C/C composites, Mater. Des. 104 (2016) $19-26$.

[13] M. Han, C.W. Zhou, H.J. Zhang, A mesoscale beam-spring combined mechanical model of needle-punched carbon/carbon composite, Compos. Sci. Technol. 168 (2018) 371-380.

[14] G.J. Weng, Some elastic properties of reinforced solids, with special reference to isotropic ones containing spherical inclusion, Int. J. Eng. Sci. 22 (1984) 845-856.

[15] S. Mortazavian, A. Fatemi, Effects of fiber orientation and anisotropy on tensile strength and elastic modulus of short fiber reinforced polymer composites, Composites Part B 72 (2015) 116-129.

[16] G.C. Oi, S.Y. Du, B.M. Zhang, Z.W. Tang, Y.L. Yu, Evaluation of carbon fibre/epoxy interfacial strength in transverse fibre bundle composite: experiment and multiscale failure modeling, Compos. Sci. Technol. 105 (2014) 1-8.

[17] K.M. Liechti, T. Freda, On the use of laminated beams for the determination of pure and mixed-mode fracture properties of structural adhesives, J. Adhes. 28 (1989) 145-169.

[18] J.C. Brewer, P.A. Lagace, Quadratic stress criterion for initiation of delamination, J. Compos. Mater. 22 (1988) 1141-1155.

[19] J.J. Nie, Y.D. Xu, L.T. Zhang, L.F. Cheng, J.Q. Ma, Microstructure and tensile behaviour of multiply needled $\mathrm{C} / \mathrm{SiC}$ composite fabricated by chemical vapor infiltration, J. Mater. Process. Technol. 209 (2009) 572-576.

[20] G.H. Zhou, Y.Q. Liu, L.L. He, Q.G. Guo, H.Q. Ye, Microstructure difference between core and skin of T700 carbon fibres in heat-treated carbon/carbon composites, Carbon 49 (2011) 2883-2892. 